\title{
Smart Clamp-Type Microwave Sensor for Accidental Leak Detection from Pipe Joints
}

\author{
Muhammad Akram Karimi ${ }^{1}$ and Atif Shamim ${ }^{2}$ \\ IMPACT Lab, King Abdullah University of Science and Technology (KAUST), Saudi Arabia \\ ${ }^{1}$ muhammadakram.karimi@kaust.edu.sa, ${ }^{2}$ atif.shamim@kaust.edu.sa
}

\begin{abstract}
Leakages from pipelines are low-probability but high impact events for distributors of crude/refined oil as well as drinking water. Several techniques including geological surveys, fiber optics, acoustics, pressure drop analysis and mass imbalance are in use to detect the leakages. However, all the techniques have their own drawbacks such as low sensitivity, high false alarm rate and process parameter dependencies. Pipe joints, being the most prone to leakages, have been targeted in this paper for real-time leakage monitoring. Instead of relying on indirect indicators such as vibration and pressure drop, we are proposing a new kind of microwave leak detector which can easily be clamped on a joint. The sensor utilizes a uniquely designed stripline ring resonator to detect the change in dielectric properties (caused by leakage) of a 3D printed porous substrate which is sandwiched between the two ground planes of the stripline. Main sensing element has been realized by a combination of $3 \mathrm{D}$ and inkjet printing. While protective clamp-style cover has been fabricated using lathe machining, 3-axis milling and wire EDM which not only protects the sensor from environmental effects such as rain but also helps avoiding false alarms. The proposed system has been validated in EM simulations as well as in measurements, which confirm that the system can not only detect the leak event but also can estimate its intensity. The measured sensitivity is better than $0.3 \mathrm{~mL}$ which is much higher than the existing methods.
\end{abstract}

Keywords-leakage detector system, ring resonator, 3D printing, inkjet printing, microwave sensor, oil sensor, stripline, pipe joint.

\section{INTRODUCTION}

Operational safety, environmental protection and economic loss are some of the main concerns due to which industries are looking for accurate and affordable leak detectors [1]. In challenging environments such as subsea oil production, leakages pose a serious threat to marine life.

Various methods have been in use in oil and water distribution networks, all over the world, to detect the accidental or planned (theft) leaks. The choice of a particular leak detection technology is driven by the application scenario. For example, in one technique, temperature profile is recorded along the pipeline using a fiber optical line and any temperature abnormality is an indicative of a potential leak [2]. However, the limited pipe length coverage and higher cost are the limiting factors for the broad acceptance of this method. Relatively common method of leak detection is to sense the vibration or sound wave emanating from the leak location [3] [4]. However, these methods of leak detection are highly dependent on the process parameters such as pipe diameter, material, medium and noise from the pump and traffic nearby [5].

Several software based leak detection techniques have also been experimented in which the data from the existing field instruments such as pressure, temperature and flow sensors are analysed to look for pressure/temperature changes or mass imbalance between two locations to predict a leakage. However, these techniques are only valid and reliable if the leakage size is as significant as $5-6 \%$ of the total flow rate [6].

All the existing leak detection systems (LDS) have one thing in common which is that they rely their measurements on an indirect parameter instead of the leaked medium itself. This is the reason that such LDS suffer from high false alarm rate making most of the systems practically infeasible.

Microwaves offer various methods to detect the change in material properties and have been in practice since many years [7]. For leak detection; EM waves are being used in geological surveys to detect the change in soil properties caused by the leaking fluid [8]. However, the problem with such surveys are that these are not real-time LDS. Instead, the leakage stays undiscovered until the survey is performed.

The focus of this paper is to use microwaves in an effective way to directly sense the leaked medium from the location that is most prone to the leakages. Joints are known to leak due to various reasons such as wear and tear and nut/bolt loosening due to the adoption of non-standard methods of tightening. We are proposing a real-time LDS designed to clamp onto the existing joints such as flanges in a simple two step procedure. In the first step, the main sensing element comprising of a stripline based ring resonator wrapped around a 3D printed porous material, is slipped on to the flange. This sensing ring is then protected by a clamp-type metallic enclosure which not only protects the sensor from extreme weather conditions but also helps in avoiding false alarms due to rain.

\section{DESIGN AND SimUlations}

The proposed microwave leak detector has been designed keeping in mind its compatibility with the existing joints. The sensor design has been built around a standard 1" metallic flange ${ }^{1}$. Most of the flanges are bolted together with an O-ring sandwiched between the two pieces. The space in between the flanges has a high probability of leakage. This is the location, we chose to place our low-cost printed resonator. The circular periphery of the flange is suitable for a ring-resonator implementation.

${ }^{1}$ 44685K13 part number from McMaster 
The idea is to absorb the leaked fluid from the flange into a $3 \mathrm{D}$ porous ring (3DPR) whose changing dielectric properties can be sensed by the shift in the resonance frequency of the ring resonator [9]. 3DPR is shown in pink colour in Fig. 1.

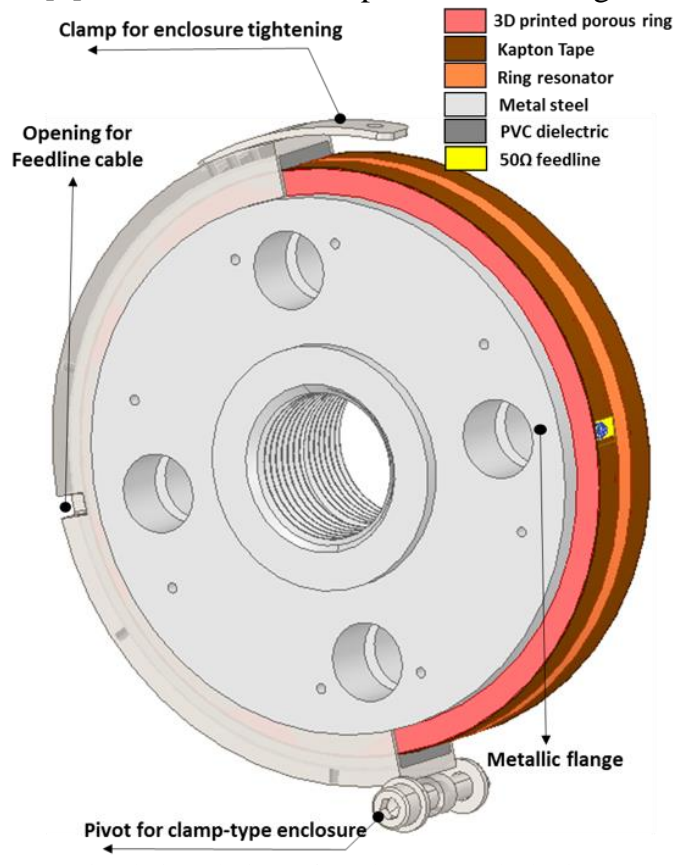

Fig. 1. HFSS simulation design of a clamp-type leak detector utilizing a stripline based ring resonator

Ring resonators are usually made on flat substrates with flat ground plane to determine the dielectric properties of the substrates. However, here we are using a ring resonator on a curved surface of a metallic flange which is being used as a ground plane. The 3DPR separates the ring resonator from the ground plane. 3DPR is filled with air in the event of no leak. As soon as the leakage of the oil or water happens; open space in 3DPR is filled up with the leaked fluid, resulting in increase of its effective dielectric constant. As 3DPR is sandwiched between the ring resonator and its ground plane (metallic flange), leakage amount can be sensed by observing the shift in resonance frequency of the ring resonator [10].

In order to protect the sensor from external effects and to shield the EM fields of the resonator; metallic enclosure has been designed which can easily be clamped on to the joint. A split view of the enclosure is shown in Fig. 1. This metallic enclosure also serves as the upper ground plane of the ringresonator while a machined piece of PVC separates the enclosure from the resonator. The flange (bottom ground) is shorted with the upper enclosure of the sensor using the side walls, as can be seen in Fig. 1. In summary, ring resonator operates in an asymmetric stripline mode whose top dielectric (PVC) is fixed in its properties while the bottom dielectric (3DPR) changes its dielectric properties as per the leakage conditions.

As per the ring-resonator theory, fundamental resonance should happen at a frequency where guided wavelength becomes equal to the mean ring circumference [9]. The ring circumference of $373 \mathrm{~mm}$ becomes equal to the guided wavelength at approximately $530-550 \mathrm{MHz}$ of frequency, which has also been proven by the simulated resonance frequency of the resonator shown in Fig. 2.

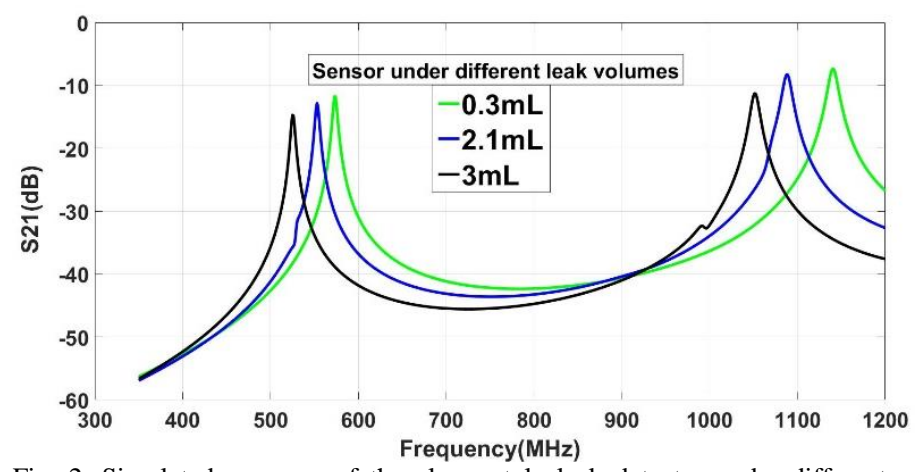

Fig. 2. Simulated response of the clamp-style leak detector under different leakage volumes of water

Moreover, at $1 \mathrm{GHz}$ frequency ( $2^{\text {nd }}$ ring resonator mode) should have four E-field maxima points along the circumference [9] which have also been validated from the simulations, as shown in Fig. 3. The part of the E-fields in the 3DPR (experiencing the changing dielectric medium, as can be seen in Fig. 3) are responsible for the change in the resonance frequency of the resonator.

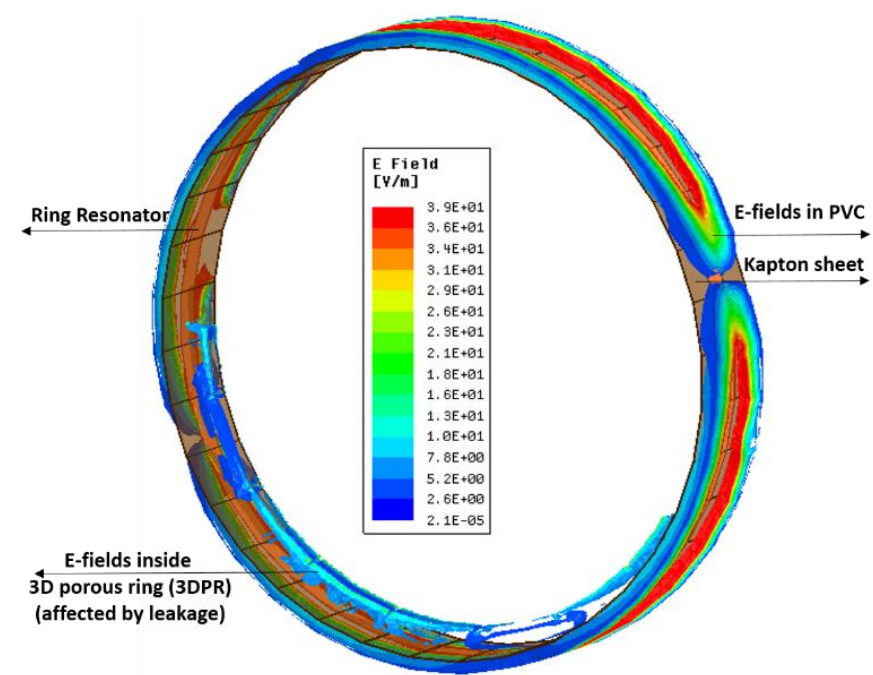

Fig. 3. E field distribution at $1 \mathrm{GHz}$ inside the fixed piece of PVC dielectric lying on top of the ring resonator as well as $3 \mathrm{D}$ porous ring (3DPR) lying underneath of it

The pores are filled up as the leaked volume from the flange increases. It results in increased effective dielectric constant (ceff) experienced by the ring-resonator. Consequently, the resonance frequency of the resonator decreases as it is inversely proportional to eeff. This has also been confirmed by the simulated results shown in Fig. 2.

It can be seen from the simulated response that the sensor resonates in the expected frequency range of $530-550 \mathrm{MHz}$ despite having an unconventional $3 \mathrm{D}$ joint-conformable clamp sensor design. 


\section{FABRICATION}

The proposed leak detection system (LDS) is effective only if it is installed over the complete fluid transmission system. This means that large numbers of such sensors will be required and thus it must be cost-effective. Due to this reason, low-cost fabrication techniques such as $3 \mathrm{D}$ and inkjet printing have been employed. The dielectric porous ring has been realized through 3D printing, as can be seen from Fig. 4, while the ring resonator has been inkjet printed on a $125 \mu \mathrm{m}$ thick kapton tape. The tape has been cleansed using ethanol followed by printing of 4 conductor layers of ANP (Ag Nano Particles) ink using 10pL cartridge. Drop spacing of $40 \mu \mathrm{m}$ has been used to achieve the desired feature size. The sample has been cured at $140^{\circ} \mathrm{C}$ for 30 minutes to achieve the desired conductivity. As can be seen from Fig. 4 that the resonator is printed as a straight line which is then wrapped around the 3DPR.

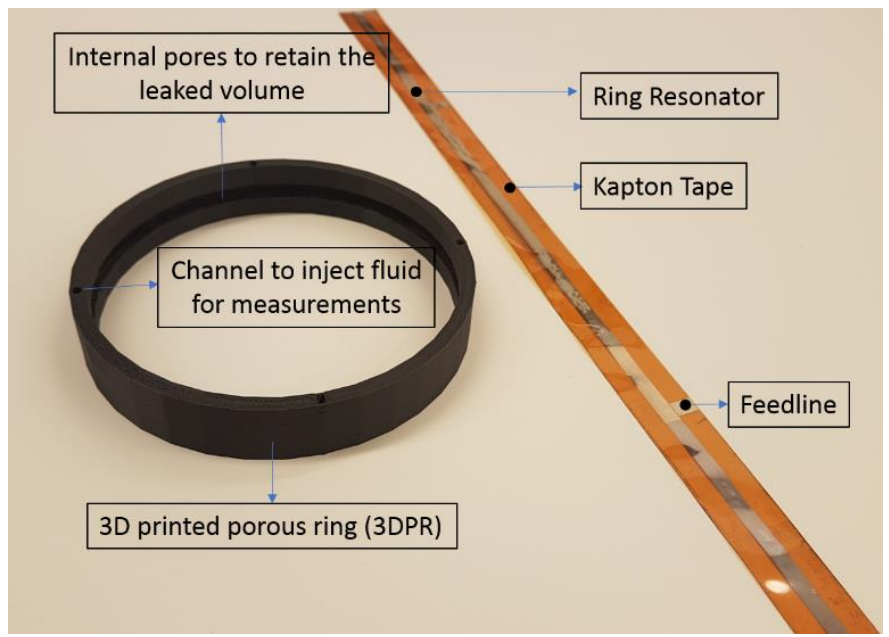

Fig. 4. 3D printed porous ring (3DPR) and kapton tape with inkjet printed ring resonator

The protective clamp-type enclosure for the sensor has been fabricated through lathe machining, 3-axis milling and wire electrical discharge machining. The sensor assembly requires a simple 2-step process as depicted in Fig. 5.

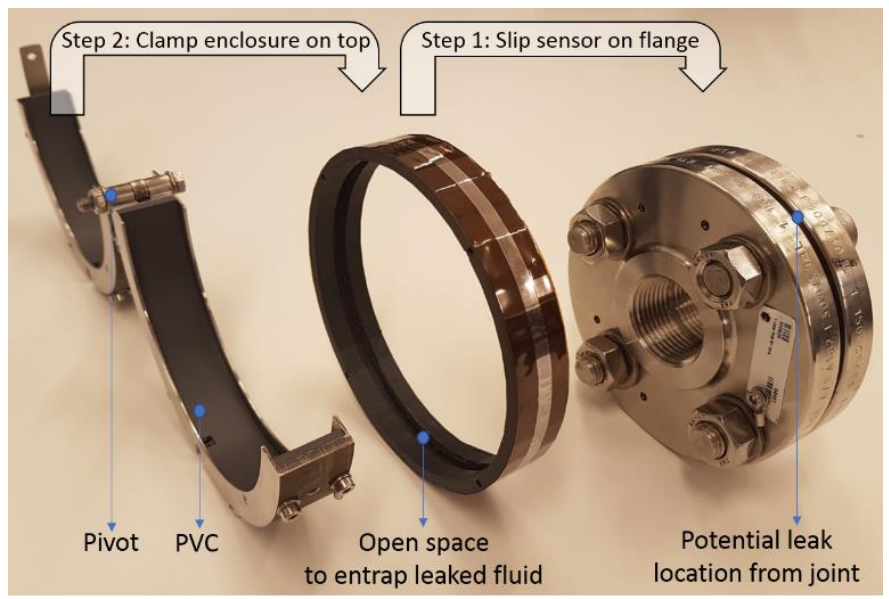

Fig. 5. Visual description of two step assembly procedure of proposed leak detector
The assembly of the leak detection system does not take more than few minutes for installation. The final assembly after following the two-step procedure is shown in Fig. 6.

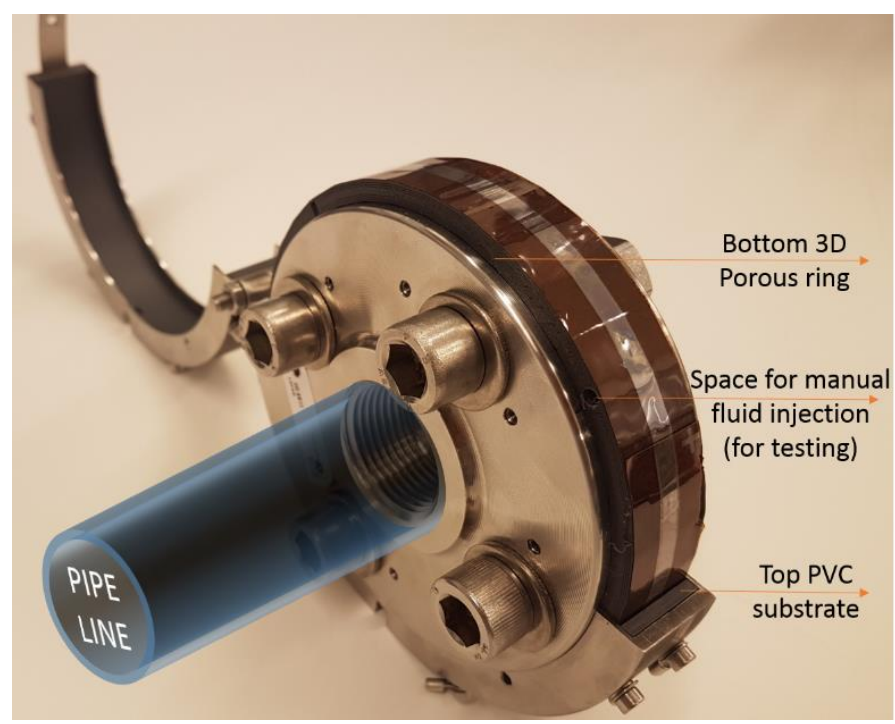

Fig. 6 . The flange assembled with proposed leak detection system

\section{MEASUREMENTS}

In an effort to make the system practically usable and as close to reality as possible, certain challenges have been faced during the measurements. For example, limited space for RF connector left us with no option other than using a $\mu \mathrm{FL}$ connector. However, it is quite challenging to make a robust bond between the connector and the curved plastic substrate while maintaining the required conductive connections. Very precise amounts of conductive and insulating epoxies have been used in a proper order for this purpose. This challenge can be overcome in a real system by making a direct connection of the resonator with an oscillator based readout circuitry.

Additionally, it has been quite challenging to manually fill the porous substrate with precise amounts of liquids. This is because the liquid volumes, being sensed, are pretty small and can also wobble inside the 3D porous ring (3DPR). Nonetheless, adopting the best possible techniques, the response of the proposed leak detector has been recorded under different leakage conditions, as shown in Fig. 7.

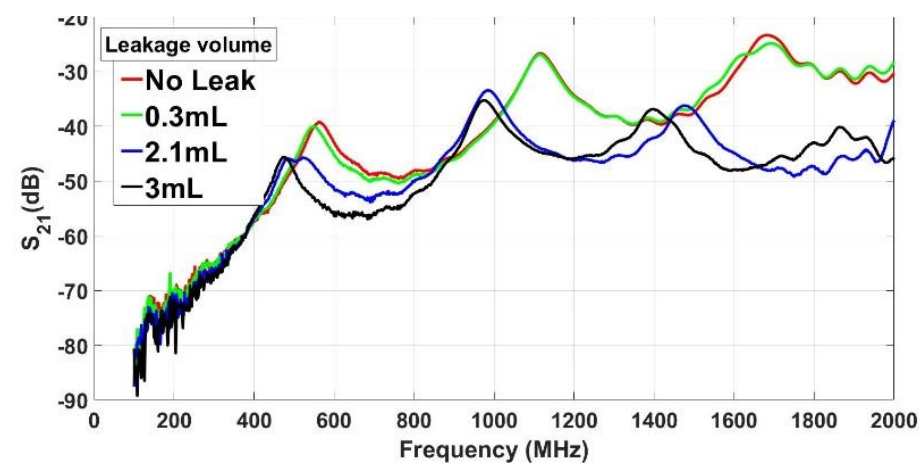

Fig. 7. Measured response of the proposed leak detector under leak conditions 
It can be seen from the comparison of red and green curves in Fig. 7, that a water leakage inside the flange, as low as $0.3 \mathrm{~mL}$, can be detected.

\section{A. Comparison of simulations with measurements}

The trends in measurements (shown in Fig. 7) are quite similar to the trends observed in simulations in Fig. 2. The resonance frequency of the ring resonator decreases with the increase in volume of the leaked fluid. The resonant frequencies of simulated and measured systems have been plotted together in Fig. 8, where it can be concluded that the proposed leak detector can not only detect very small amounts of leakages but can also predict the magnitude of the leakage, a feature that is not commonly available in the existing leak detectors. The gap between the simulated and measured resonant frequencies can be due to slightly different dielectric constants of the substrates used in the simulations compared to the real measurements.

It can also be observed from the comparison of Fig. 2 and Fig. 7, that the measured loss of the ring resonator is higher as compared to the simulations. This can potentially be because of the low conductivity of the inkjet-printed metal, which is assumed to be higher in the simulations.

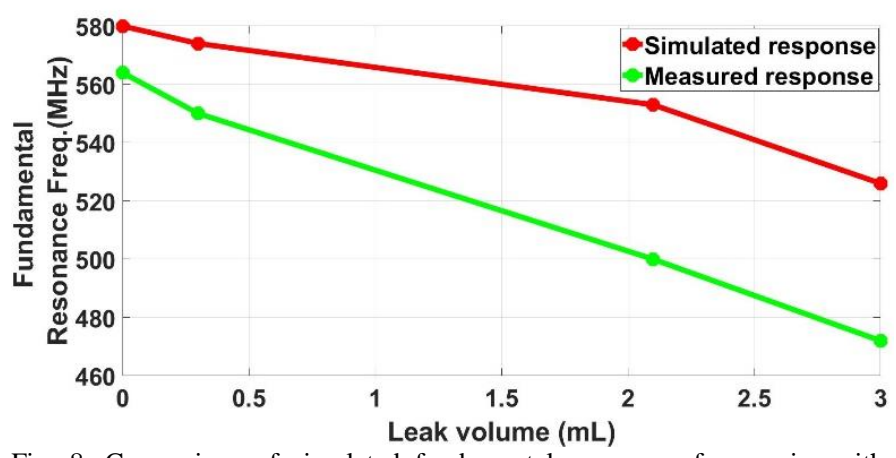

Fig. 8. Comparison of simulated fundamental resonance frequencies with measured fundamental resonance frequencies

\section{PRACTICAL USE OF THE PROPOSED SySteM}

At present, the proposed system uses readily available $3 \mathrm{D}$ printed and PVC materials in its fabrication. However, these materials can easily be replaced by some other high pressure and high temperature (HPHT) tolerant dielectric materials such as PEEK. Such materials can easily work up to high line pressures and temperatures as high as $125^{\circ} \mathrm{C}$. All other materials used, such as steel-304, kapton and silver ink can withstand high temperatures. Thus, it is expected that very little modification will be required in the packaging of the sensor before it can be used for practical applications.

\section{CONCLUSION}

For the first time ever, we have demonstrated a leakage detection system which measures the actual leaked medium instead of relying on any indirect readings. The presented approach gives a leakage sensitivity as high as $300 \mu \mathrm{L}$ which is not achievable by any indirect measurement technique. The presented design is easily scalable for higher pipe sizes by scaling-up the size of the resonator. In future, a microwave oscillator can be integrated with the resonator to detect any changes in the resonance frequency. This will eliminate the need of any measurement equipment such as VNA and thus a self-contained leak detection system can be developed for smart joints of the future.

\section{REFERENCES}

[1] T. Slade, Y. Okamoto and J. Talor, "Economic Benefits of Leak Detection Systems: A Quantitative Methodology," in PSIG Annual Meeting, Baltimore, 2014.

[2] S. K. R. S. Lawrence BOAZ Kalubu, "An overview of pipeline leak detection and location systems," in Pan African International Conference on Information Science, Computing and Telecommunications, 2014.

[3] J. Choi, J. Shin, C. Song, S. Han and D. I. Park, "Leak Detection and Location ofWater Pipes Using Vibration Sensors and Modified ML Prefilter," Sensors, 2017.

[4] J. Wan, Y. Yu, Y. Wu, R. Feng and N. Yu, "Hierarchical Leak Detection and Localization Method in Natural Gas Pipeline Monitoring Sensor Networks," Sensors, vol. 12, no. 1, pp. 189-214, 2012.

[5] O. Hunaidi, "Detecting Leaks in Water-Distribution Pipes," 2000. [Online].

[6] Emerson, "Best Practices in Leak and Theft Detection," April 2016. [Online].

[7] M. A. Karimi and A. S. Muhammad Arsalan, "Design and Dynamic Characterization of an Orientation Insensitive Microwave Water-Cut Sensor," IEEE TRANSACTIONS ON MICROWAVE THEORY AND TECHNIQUES, vol. 66, no. 1, pp. 530-539, 2018.

[8] HydroGeophysics, "Pipelines," HydroGeophysics, [Online]. Available: https://www.hgiworld.com/services/leak-detection-2/pipe/. [Accessed 01 December 2018].

[9] A. A. Abduljabar, D. J. Rowe, A. Porch and D. A. Barrow, "Novel Microwave Microfluidic Sensor Using a Microstrip Split-Ring Resonator," IEEE Transactions on Microwave Theory and Techniques, vol. 62, no. 3, pp. 679-688, 2014.

[10] M. A. Karimi, M. Arsalan and A. Shamim, "A low cost, printed microwave based level sensor with integrated oscillator readout circuitry," in 2017 IEEE MTT-S International Microwave Symposium (IMS), Honololu, 2017. 